\title{
Fourth-order interference in the Wigner representation for parametric down-conversion experiments
}

\author{
Alberto Casado, ${ }^{1, *}$ Agustín Fernández-Rueda, ${ }^{1, *}$ Trevor Marshall, ${ }^{2}$ Ramón Risco-Delgado, ${ }^{1, *}$ and Emilio Santos ${ }^{1}$ \\ ${ }^{1}$ Departamento de Física Moderna, Universidad de Cantabria, 39005 Santander, Spain \\ ${ }^{2}$ Department of Mathematics, University of Manchester, Manchester M13 9PL, United Kingdom
}

(Received 17 July 1996)

\begin{abstract}
In the Wigner formalism, after giving a general description of a light beam, the theory of parametric down-conversion is developed to second-order in the coupling constant. We then describe the detection process by calculating the auto-correlation and cross-correlations of the signal and idler beams. Four recent experiments are analyzed in detail: interference on a screen, Franson's experiments [Phys. Rev. Lett. 62, 2205 (1989)], Rarity and Tapster's experiments [Phys. Rev. Lett. 64, 2495 (1990)], and induced coherence without induced emission [X. Y. Zou, L. J. Wang, and L. Mandel, Phys. Rev. Lett. 67, 318 (1991)].
\end{abstract} [S1050-2947(97)03005-9]

PACS number(s): 42.50.Ar, 03.65.Sq, 42.50.Lc

\section{INTRODUCTION}

Experiments using photon pairs from parametric downconversion (PDC) have become very popular in the past decade for the study of nonclassical aspects of light [1]. In the pioneer experiment of Burnham and Weinberg [2], it was found that the measured value of the correlation time between the two down-converted photons was very small, and more recent experiments have shown that it may be smaller than picoseconds [3]. This means that experiments with PDC photons pairs are well suited for the study of the quantum aspects of light, such as photon entanglement.

The theory of PDC has been developed using the standard Hilbert-space formulation of quantum optics [4], but, to our knowledge, no study of PDC has been made using the phasespace distributions, which are so popular in other parts of quantum optics. For instance, phase-space distributions provide the standard method for the study of parametric amplification [5], closely related to PDC. Obviously, the reason is that the Hilbert-space formulation seems more suitable when the photon number is the relevant observable, as in PDC, while phase-space representations seem better when the field amplitudes are the relevant quantities, as in the case of parametric amplification. Here we shall show that the Wigner representation is also quite efficient for analyzing experiments involving PDC photon pairs.

Furthermore, this formulation stresses the role played by the vacuum fields incident on the crystal [6]. Quantum theory does not allow for a picture with entities (either pure particles or pure waves) propagating in space and time. Nevertheless, the Hilbert-space formulation emphasizes the particle aspect. Photons are created at some point, propagate, and are eventually annihilated by detectors. Of course the photons are not classical particles and, for instance, their

\footnotetext{
*Permanent address: Departamento de Física Aplicada, Escuela Superior de Ingenieros, Universidad de Sevilla, Sevilla, Spain. Electronic address: acasado@cica.es
}

possible trajectories seem to interfere. In contrast, the phasespace representations suggest an interpretation in terms of waves. If the Wigner distribution is interpreted as a probability distribution of field-mode amplitudes, then the corpuscular aspect of light appears just as wave interference, including a zero-point vacuum field. There are two difficulties for this interpretation: (i) The Wigner function associated with the quantum states of light is not always non-negative definite and, consequently, it cannot always be interpreted as a probability distribution, and (ii) the detection probability is not proportional to the intensity, but to the difference between the actual intensity and the zero-point intensity (see Sec. V). In PDC experiments the first difficulty does not appear because the Wigner function is always positive in these experiments (see Sec. IV).

In the rest of the paper we shall study in the Wigner representation the essential ingredients in order to interpret the experiments. In Sec. II we describe the light beam. In Sec. III an explicit expression for the Wigner fields produced in the process of PDC is obtained. Section IV is devoted to the study of correlations among these beams as a consequence of the correlations present in the vacuum field. Expressions for the single and joint detection probabilities are computed in Sec. V. In Secs. VI-IX we study a representative set of the experiments.

In a previous paper [7] we have studied in the Wigner representation several experiments with parametric downconverted light involving single counts. These experiments showed second-order interference. In the present paper we study experiments that show fourth-order interference and therefore involve coincidence detections.

\section{DESCRIPTION OF A LIGHT BEAM IN THE WIGNER REPRESENTATION}

In the Hilbert-space representation of the light field, the electric vector is represented as a sum of two mutually conjugate operators

$$
\hat{\mathbf{E}}(\mathbf{r}, t)=\hat{\mathbf{E}}^{(+)}(\mathbf{r}, t)+\hat{\mathbf{E}}^{(-)}(\mathbf{r}, t),
$$




$$
\hat{\mathbf{E}}^{(+)}(\mathbf{r}, t)=i \sum_{\mathbf{k}, \lambda}\left(\frac{\hbar \omega_{\mathbf{k}}}{2 L^{3}}\right)^{1 / 2} \boldsymbol{\epsilon}_{\mathbf{k}, \lambda} \hat{a}_{\mathbf{k}, \lambda}(t) e^{i \mathbf{k} \cdot \mathbf{r}}
$$

where $L^{3}$ is the normalization volume and $\hat{a}_{\mathbf{k}, \lambda}(t)$ is the annihilation operator for a photon whose wave vector is $\mathbf{k}$ and whose polarization vector is $\boldsymbol{\epsilon}_{\mathbf{k}, \lambda}$, and $\omega_{\mathbf{k}}=c|\mathbf{k}|$. Equations (1) and (2) correspond to the Heisenberg picture, where all time dependence goes in the creation and annihilation operators $\hat{a}_{\mathbf{k}, \lambda}^{\dagger}(t)$ and $\hat{a}_{\mathbf{k}, \lambda}(t)$. For a free field this dependence has the form

$$
\hat{a}_{\mathbf{k}, \lambda}(t)=\hat{a}_{\mathbf{k}, \lambda}(0) e^{-i \omega_{\mathbf{k}} t},
$$

but for interacting fields it is complicated and contains all the dynamics of the process. In this picture the state of the field is represented by a time-independent density operator $\rho$.

In this paper we shall always consider electromagnetic fields corresponding to narrow light beams and we shall not study experiments involving polarizing devices. In these conditions it is convenient to use a scalar approximation well known in classical optics. We assume that the light beam contains frequencies within a range between $\omega_{\min }$ and $\omega_{\max }$ and wave vectors whose transverse components are limited by a small upper value, that is,

$$
\omega_{\min }<\omega_{\mathbf{k}}<\omega_{\max }, \quad\left|\mathbf{k}^{\mathrm{tr}}\right| \ll \frac{\omega_{\min }}{c} .
$$

We shall ignore polarization and therefore multiply the amplitude (2) by $\sqrt{2}$. Hence the "relevant" component of the electric vector is

$$
\hat{E}^{(+)}(\mathbf{r}, t)=i \sum_{[\mathbf{k}]}\left(\frac{\hbar \omega_{\mathbf{k}}}{L^{3}}\right)^{1 / 2} \hat{a}_{\mathbf{k}}(t) e^{i \mathbf{k} \cdot \mathbf{r}}
$$

where the square brackets in the summation symbol indicate that the sum is restricted to the set of $\mathbf{k}$ satisfying Eq. (4).

In the Wigner representation the operators $\hat{E}^{(+)}(\mathbf{r}, t)$ and $\hat{E}^{(-)}(\mathbf{r}, t)$ become $c$ numbers, the annihilation operators $\hat{a}_{\mathbf{k}}(t)$ being replaced by random variables $\alpha_{\mathbf{k}}(t)$ and their Hermitian conjugates $\hat{a}_{\mathbf{k}}^{\dagger}(t)$ by complex conjugates $\alpha_{\mathbf{k}}^{*}(t)$. The field amplitudes are

$$
E^{(+)}(\mathbf{r}, t)=i \sum_{[\mathbf{k}]}\left(\frac{\hbar \omega_{\mathbf{k}}}{L^{3}}\right)^{1 / 2} \alpha_{\mathbf{k}}(t) e^{i \mathbf{k} \cdot \mathbf{r}} .
$$

The Wigner density associated with a state whose density matrix is $\rho$ is [8]

$$
W\left(\alpha, \alpha^{*}\right)=\operatorname{Tr}\left[\rho \Phi\left(\alpha, \alpha^{*}\right)\right] \quad\left(\alpha \equiv\left\{\alpha_{\mathbf{k}}\right\}\right),
$$

where

$$
\Phi\left(\alpha, \alpha^{*}\right)=\prod_{[\mathbf{k}]} \frac{1}{\pi^{2}} \int e^{\beta_{\mathbf{k}}\left(\hat{a}_{\mathbf{k}}^{\dagger}-\alpha_{\mathbf{k}}^{*}\right)-\beta_{\mathbf{k}}^{*}\left(\hat{a}_{\mathbf{k}}-\alpha_{\mathbf{k}}\right)} d^{2} \beta_{\mathbf{k}} .
$$

For instance, the Wigner function of the vacuum state is the Gaussian

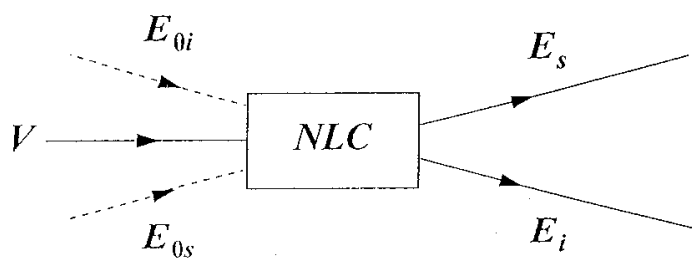

FIG. 1. The process of parametric down-conversion.

$$
W_{\mathrm{vac}}\left(\alpha, \alpha^{*}\right)=\prod_{[\mathbf{k}]} \frac{2}{\pi} e^{-2\left|\alpha_{\mathbf{k}}\right|^{2}}
$$

The crucial property of the Wigner function is that the ensemble average of any polynomial of the random variables $\alpha$ and $\alpha^{*}$ weighted by the Wigner density exactly corresponds to the Hilbert space expectation of the corresponding symmetrized product of the annihilation and creation operators $\hat{a}$ and $\hat{a}^{\dagger}$. That is,

$$
\begin{aligned}
\left\langle P\left(\alpha, \alpha^{*}\right)\right\rangle & =\int P\left(\alpha, \alpha^{*}\right) W\left(\alpha, \alpha^{*}\right) d^{2 M} \alpha \\
& =\operatorname{Tr}\left\{\rho S\left[P\left(\hat{a}, \hat{a}^{\dagger}\right)\right]\right\},
\end{aligned}
$$

where $M$ is the number of $\alpha_{\mathbf{k}}$ variables defined and $S[]$ means symmetrization, which consists of taking the average over all orderings of the operators, for example,

$$
S\left[\hat{E}^{(+)} \hat{E}^{(-)}\right]=\frac{1}{2}\left(\hat{E}^{(+)} \hat{E}^{(-)}+\hat{E}^{(-)} \hat{E}^{(+)}\right) .
$$

Another useful piece of information is the transformation of the Wigner field amplitudes in a beam splitter (BS). If $a, b$ are the incoming channels and $c, d$ the outgoing ones, and $T(R)$ is the transmission (reflection) coefficient, we have

$$
\begin{aligned}
& E_{c}^{(+)}(\mathbf{r}, t)=T E_{a}^{(+)}(\mathbf{r}, t)+i R E_{b}^{(+)}(\mathbf{r}, t), \\
& E_{d}^{(+)}(\mathbf{r}, t)=T E_{b}^{(+)}(\mathbf{r}, t)+i R E_{a}^{(+)}(\mathbf{r}, t) .
\end{aligned}
$$

We have assumed that $R$ and $T$ are real numbers and $\mathbf{r}$ is the point where the center of the BS is placed. These relations are the same as between the corresponding field operators in the Hilbert-space formalism and also between field amplitudes in classical optics. This agreement is a straightforward consequence of the linearity of Eqs. (12).

\section{THE PROCESS OF PARAMETRIC DOWN-CONVERSION}

In this section we are going to study the process of parametric down-conversion of light in the Wigner representation. In Fig. 1 we show a sketch of the setup used for PDC. A nonlinear crystal is pumped by a laser beam $V$, giving rise to a rainbow of colored cones around the axis defined by the pump. In experimental practice two narrow correlated beams, called "signal" $E_{s}$ and "idler" $E_{i}$, are selected by means of pinholes, filters, or just the detectors.

The process of PDC may be formalized using a Hamiltonian of the form 


$$
\hat{H}=\sum_{\mathbf{k}} \hbar \omega_{\mathbf{k}}\left(\hat{a}_{\mathbf{k}}^{\dagger} \hat{a}_{\mathbf{k}}+\frac{1}{2}\right)+\hat{H}_{\mathrm{int}},
$$

where

$$
\hat{H}_{\mathrm{int}}=i \hbar g^{\prime} V \sum_{\mathbf{k}, \mathbf{k}^{\prime}} f\left(\mathbf{k}, \mathbf{k}^{\prime}\right) e^{-i \omega_{0} t} \hat{a}_{\mathbf{k}}^{\dagger} \hat{a}_{\mathbf{k}^{\prime}}^{\dagger}+\text { H.c. },
$$

and we have taken the origin of the coordinate system at the center of the crystal. In Eq. (14) we have treated the pump beam as an intense monochromatic plane wave represented, in the scalar approximation, by

$$
V(\mathbf{r}, t)=V e^{i\left(\mathbf{k}_{0} \cdot \mathbf{r}-\omega_{0} t\right)}+\text { c.c. }
$$

It is not quantized because it is much more intense than the outgoing beams. The coupling constant $g^{\prime}$ is defined so that the product $g^{\prime} V$ has dimensions of frequency, and $f\left(\mathbf{k}, \mathbf{k}^{\prime}\right)$ is a dimensionless symmetrical function of the wave vectors inside the crystal. This function, which is related to the function $f(\mathbf{k}, s ; \mathbf{r}, t)$ introduced in Eq. (15) of Ref. [4], is different from zero only when the following matching condition is fulfilled:

$$
\mathbf{k}_{0} \approx \mathbf{k}+\mathbf{k}^{\prime}
$$

As is well known [4], there is in addition a matching condition for frequency that is fulfilled much more rigorously, namely,

$$
\omega_{0}=\omega_{\mathbf{k}}+\omega_{\mathbf{k}^{\prime}}
$$

We now obtain the Heisenberg equations of motion for $\hat{a}_{\mathbf{k}}(t)$ during the interaction, using the Hamiltonian given by Eq. (13),

$$
\begin{gathered}
\dot{\hat{a}}_{\mathbf{k}}=\frac{1}{i \hbar}\left[\hat{a}_{\mathbf{k}}, \hat{H}\right] \rightarrow \\
\dot{\hat{a}}_{\mathbf{k}}=-i \omega_{\mathbf{k}} \hat{a}_{\mathbf{k}}+g^{\prime} V \sum_{\mathbf{k}^{\prime}} f\left(\mathbf{k}, \mathbf{k}^{\prime}\right) e^{-i \omega_{0} t} \hat{a}_{\mathbf{k}^{\prime}}^{\dagger} .
\end{gathered}
$$

In order to calculate $\hat{a}_{\mathbf{k}}(t)$ for all $t$ we shall take into account that the operator $\hat{a}_{\mathbf{k}}(t)$ evolves as a free-field mode before entering the crystal and after coming out. We shall integrate Eq. (18) from $t=-\Delta t$ to $t=0$, where $\Delta t$ is the time taken for the radiation to cross the crystal. The initial condition is $\hat{a}_{\mathbf{k}}(-\Delta t)=\hat{a}_{0 \mathbf{k}}(-\Delta t)$, where $\hat{a}_{0 \mathbf{k}}(-\Delta t)$ is the destruction operator of the mode $\mathbf{k}$ in the incoming vacuum field. We shall assume that the coherence time of the laser is large in comparison with most of the times involved in the process, so that we may ignore the time dependence of $V(t)$. Because the detection probabilities are of second-order in the coupling constant $g^{\prime}$, we need, in general, to calculate $\hat{a}_{\mathbf{k}}(t)$ to second-order in $g^{\prime}$. This fact plays an essential role in the calculation of probabilities in the Wigner representation. However, we shall show that where, as in this article, only joint probabilities are calculated, all second-order terms may be expressed in terms of first-order ones.
To second-order in the coupling constant $g^{\prime}$, that is, taking the second term of the right-hand side of Eq. (18) as a perturbation and retaining terms up to order $g^{\prime 2}$, we get (setting $g^{\prime} \Delta t \equiv g$ )

$$
\begin{aligned}
\hat{a}_{\mathbf{k}}(0)= & \hat{a}_{0 \mathbf{k}}(0)+g V \sum_{\mathbf{k}^{\prime}} f\left(\mathbf{k}, \mathbf{k}^{\prime}\right) u\left[\frac{\Delta t}{2}\left(\omega_{0}-\omega_{\mathbf{k}}-\omega_{\mathbf{k}^{\prime}}\right)\right] \\
& \times \hat{a}_{0 \mathbf{k}^{\prime}}^{\dagger}(0)+g^{2}|V|^{2} \sum_{\mathbf{k}^{\prime}} \sum_{\mathbf{k}^{\prime \prime}} f\left(\mathbf{k}, \mathbf{k}^{\prime}\right) f^{*}\left(\mathbf{k}^{\prime}, \mathbf{k}^{\prime \prime}\right) \\
& \times u\left[\frac{\Delta t}{2}\left(\omega_{\mathbf{k}^{\prime}}+\omega_{\mathbf{k}^{\prime \prime}}-\omega_{0}\right)\right] \\
& \times u\left[\frac{\Delta t}{2}\left(\omega_{\mathbf{k}^{\prime \prime}}-\omega_{\mathbf{k}}\right)\right] \hat{a}_{0 \mathbf{k}^{\prime \prime}}(0)
\end{aligned}
$$

where

$$
u(x)=\frac{\sin x}{x} e^{i x} .
$$

After $t=0, \hat{a}_{\mathbf{k}}(t)$ evolves as a free-field mode

$$
\hat{a}_{\mathbf{k}}(t)=\hat{a}_{\mathbf{k}}(0) e^{-i \omega_{\mathbf{k}} t}
$$

In the derivation of Eq. (19) we have considered the free evolution of operators $\hat{a}_{0 \mathbf{k}}$ (zeroth-order solution)

$$
\hat{a}_{0 \mathbf{k}}(0)=\hat{a}_{0 \mathbf{k}}(-\Delta t) e^{-i \omega_{\mathbf{k}} \Delta t}, \quad \hat{a}_{0 \mathbf{k}}^{\dagger}(0)=\hat{a}_{0 \mathbf{k}}^{\dagger}(-\Delta t) e^{i \omega_{\mathbf{k}} \Delta t} .
$$

The perturbative approximation used to get Eq. (19) is valid provided that

$$
g|V| \ll 1
$$

From now on we shall use $g$, instead of $g^{\prime}$, as an effective coupling constant. Equation (16) implies $\mathbf{k}^{\prime \prime} \approx \mathbf{k}$ in the second-order contribution to Eq. (19). Finally, taking into account that commutation rules are preserved in a unitary evolution, it is not difficult to see that [from Eq. (21)]

$$
\begin{gathered}
{\left[\hat{a}_{\mathbf{k}}\left(t_{1}\right), \hat{a}_{\mathbf{k}^{\prime}}\left(t_{2}\right)\right]=0,} \\
{\left[\hat{a}_{\mathbf{k}}\left(t_{1}\right), \hat{a}_{\mathbf{k}^{\prime}}^{\dagger}\left(t_{2}\right)\right]=\delta_{\mathbf{k}, \mathbf{k}^{\prime}} e^{-i \omega_{\mathbf{k}}\left(t_{1}-t_{2}\right)}, \quad t_{1}, t_{2}>0 .}
\end{gathered}
$$

Commutators (24) will be used in Sec. IV, in order to relate different correlations.

In order to go to the Wigner representation, we shall use the fact that the evolution equations of the Wigner field amplitudes are the same as Heisenberg equations of motion of the corresponding quantum field amplitudes, whenever the latter are linear. The linearity of the Heisenberg equations is a consequence of the fact that the Hamiltonian (14) is quadratic in the creation and/or annihilation operators. Hence we simply replace operators $\hat{a}_{\mathbf{k}}\left(\hat{a}_{\mathbf{k}}^{\dagger}\right)$ by complex variables $\alpha_{\mathbf{k}}$ $\left(\alpha_{\mathbf{k}}^{*}\right)$ in expressions (19) and (21).

Now, let us consider two narrow correlated beams, called signal and idler, with average frequencies $\omega_{s}, \omega_{i}$ and wave vectors $\mathbf{k}_{s}, \mathbf{k}_{i}$, respectively, fulfilling the matching conditions 


$$
\omega_{s}+\omega_{i}=\omega_{0}, \quad \mathbf{k}_{s}+\mathbf{k}_{i}=\mathbf{k}_{0} .
$$

From expressions (19), (21) (as functions of $\alpha, \alpha^{*}$ ), and (6), we obtain

$$
\begin{aligned}
E_{s}^{(+)}(\mathbf{r}, t)= & E_{0 s}^{(+)}(\mathbf{r}, t)+e^{-i \omega_{0} t} g V G E_{0 i}^{(-)}(\mathbf{r}, t) \\
& +g^{2}|V|^{2} J E_{0 s}^{(+)}(\mathbf{r}, t), \\
E_{i}^{(+)}(\mathbf{r}, t)= & E_{0 i}^{(+)}(\mathbf{r}, t)+e^{-i \omega_{0} t} g V G E_{0 s}^{(-)}(\mathbf{r}, t) \\
& +g^{2}|V|^{2} J E_{0 i}^{(+)}(\mathbf{r}, t) .
\end{aligned}
$$

Here $E_{0 s}$ and $E_{0 i}$ are the incoming vacuum field and $E_{s}$ $\left(E_{i}\right)$ the outgoing signal (idler); see Fig. 1. We have

$$
E_{0 s}^{(+)}(\mathbf{r}, t)=i \sum_{[\mathbf{k}]_{s}}\left(\frac{\hbar \omega_{\mathbf{k}}}{L^{3}}\right)^{1 / 2} e^{i\left(\mathbf{k} \cdot \mathbf{r}-\omega_{\mathbf{k}} t\right)} \alpha_{0 \mathbf{k}}(0),
$$

where the square brackets in the summation symbol indicate that the sum is restricted to the set of $\mathbf{k}$ pertaining to the signal beam, and similarly for $E_{0 i}^{(+)}$. For brevity we have introduced the linear operators $G$ and $J$, which are defined as

$$
G E_{0 i}^{(-)}(\mathbf{r}, t)=i \sum_{[\mathbf{k}]_{s}}\left(\frac{\hbar \omega_{\mathbf{k}}}{L^{3}}\right)^{1 / 2} e^{i \mathbf{k} \cdot \mathbf{r}} e^{i\left(-\omega_{0}-\omega_{\mathbf{k}}\right) t} \beta_{\mathbf{k}},
$$

with

$$
\beta_{\mathbf{k}}=\sum_{\left[\mathbf{k}^{\prime}\right]_{i}} f\left(\mathbf{k}, \mathbf{k}^{\prime}\right) u\left[\frac{\Delta t}{2}\left(\omega_{0}-\omega_{\mathbf{k}}-\omega_{\mathbf{k}^{\prime}}\right)\right] \alpha_{0 \mathbf{k}^{\prime}}^{*}(0),
$$

and

$$
J E_{0 s}^{(+)}(\mathbf{r}, t)=i \sum_{[\mathbf{k}]_{s}}\left(\frac{\hbar \omega_{\mathbf{k}}}{L^{3}}\right)^{1 / 2} e^{i \mathbf{k} \cdot \mathbf{r}} e^{-i \omega_{\mathbf{k}} t} \gamma_{\mathbf{k}},
$$

with

$$
\begin{aligned}
\gamma_{\mathbf{k}}= & \sum_{\left[\mathbf{k}^{\prime}\right]_{i}} \sum_{\left[\mathbf{k}^{\prime \prime}\right]_{s}} f\left(\mathbf{k}, \mathbf{k}^{\prime}\right) f^{*}\left(\mathbf{k}^{\prime}, \mathbf{k}^{\prime \prime}\right) u\left[\frac{\Delta t}{2}\left(\omega_{\mathbf{k}^{\prime}}+\omega_{\mathbf{k}^{\prime \prime}}-\omega_{0}\right)\right] \\
& \times u\left[\frac{\Delta t}{2}\left(\omega_{\mathbf{k}^{\prime \prime}}-\omega_{\mathbf{k}}\right)\right] \alpha_{0 \mathbf{k}^{\prime \prime}}(0) .
\end{aligned}
$$

From Eq. (26) we see that the outgoing signal, to order $g^{2}$, consists of three parts: (i) a zero-point radiation with amplitude $E_{0 s}^{(+)}$, which passes through the crystal without any change; (ii) a radiation produced by the nonlinear interaction (mediated by the crystal) between the laser beam, with amplitude $V$, and the zero-point radiation, with amplitude $E_{0 i}^{(-)}$, entering the crystal in the direction of the idler beam; and (iii) one part that modifies the amplitude $E_{0 s}^{(+)}$just a little (to order $g^{2}$ ). The idler beam is constituted in a similar manner.

\section{SIGNAL AND IDLER FIELD AUTOCORRELATIONS AND CROSS CORRELATIONS}

In order to compute the detection probabilities in the Wigner representation, we now calculate the correlation properties of the fields. From Eqs. (26) and (27) we see that, in the Wigner representation, the signal and idler beams are linearly related to the incoming vacuum. Therefore, they are Gaussian stochastic processes of zero mean. The reason is that the vacuum amplitudes are Gaussian [see Eq. (9)] and this property is preserved by linear transformations. In the Heisenberg picture that we are using, the Wigner distribution is time independent and given by Eq. (9), which is positive. If we pass to the Schrödinger picture the linearity of the evolution equations (18) implies that the Wigner function remains positive at all times.

In the work that follows it proves convenient to substitute slowly varying amplitudes $F^{(+)}(\mathbf{r}, t)\left[F^{(-)}(\mathbf{r}, t)\right]$ for the amplitudes $E^{(+)}(\mathbf{r}, t)\left[E^{(-)}(\mathbf{r}, t)\right]$ defined in Eq. (6), the relation between them being

$$
\begin{aligned}
F^{(+)}(\mathbf{r}, t) & =e^{i \omega_{a} t} E^{(+)}(\mathbf{r}, t) \\
& =i \sum_{[\mathbf{k}]}\left(\frac{\hbar \omega_{\mathbf{k}}}{L^{3}}\right)^{1 / 2} \alpha_{\mathbf{k}}(0) e^{i \mathbf{k} \cdot \mathbf{r}} e^{i\left(\omega_{a}-\omega_{\mathbf{k}}\right) t},
\end{aligned}
$$

where $\omega_{a}$ is some appropriately chosen average frequency midway between $\omega_{\min }$ and $\omega_{\max }$ [see Eq. (4)].

In the study of the experiments of the following sections the dependence of the field amplitudes on position will be essential. It is easy to obtain the amplitude $F^{(+)}\left(\mathbf{r}_{B}, t\right)$ in terms of the amplitude $F^{(+)}\left(\mathbf{r}_{A}, t\right)$ at another point of the light beam. Using the scalar approximation of the electric field, we readily have

$$
F^{(+)}\left(\mathbf{r}_{B}, t\right)=F^{(+)}\left(\mathbf{r}_{A}, t-\frac{r_{A B}}{c}\right) e^{i \omega_{a}\left(r_{A B} / c\right)},
$$

where $\mathbf{r}_{A B}=\mathbf{r}_{B}-\mathbf{r}_{A}$ and $r_{A B}=\left|\mathbf{r}_{A B}\right|$.

We point out that the expressions for the detection probabilities (see Sec. V) remain valid when the slowly varying amplitudes $F^{(+)}$or $F^{(-)}$are substituted for the usual ones $E^{(+)}$or $E^{(-)}$. Consequently, we shall use only the amplitudes $F^{(+)}$and $F^{(-)}$in the rest of this paper.

In terms of the slowly varying amplitudes, Eqs. (26) and (27) may be written

$$
\begin{aligned}
& F_{s}^{(+)}(\mathbf{r}, t)=\left(1+g^{2}|V|^{2} J\right) F_{0 s}^{(+)}(\mathbf{r}, t)+g V G F_{0 i}^{(-)}(\mathbf{r}, t), \\
& F_{i}^{(+)}(\mathbf{r}, t)=\left(1+g^{2}|V|^{2} J\right) F_{0 i}^{(+)}(\mathbf{r}, t)+g V G F_{0 s}^{(-)}(\mathbf{r}, t),
\end{aligned}
$$

where

$$
F_{0 s}^{(+)}(\mathbf{r}, t)=i \sum_{[\mathbf{k}]_{s}}\left(\frac{\hbar \omega_{\mathbf{k}}}{L^{3}}\right)^{1 / 2} e^{i \mathbf{k} \cdot \mathbf{r}} e^{i\left(\omega_{s}-\omega_{\mathbf{k}}\right) t} \alpha_{0 \mathbf{k}}(0)
$$

and similarly for $F_{0 i}^{(+)}(\mathbf{r}, t)$.

\section{A. Autocorrelations}

For the field at a given point $\mathbf{r}$ and two different times $t$ and $t^{\prime}$ we have to order $g^{2}$

$$
\begin{aligned}
\left\langle F_{s}^{(+)}\right. & \left.(\mathbf{r}, t) F_{s}^{(-)}\left(\mathbf{r}, t^{\prime}\right)\right\rangle-\left\langle F_{0 s}^{(+)}(\mathbf{r}, t) F_{0 s}^{(-)}\left(\mathbf{r}, t^{\prime}\right)\right\rangle \\
= & g^{2}|V|^{2}\left\{\left\langle G F_{0 i}^{(-)}(\mathbf{r}, t) G^{*} F_{0 i}^{(+)}\left(\mathbf{r}, t^{\prime}\right)\right\rangle+\left\langle F_{0 s}^{(+)}(\mathbf{r}, t) J^{*}\right.\right. \\
& \left.\left.\times F_{0 s}^{(-)}\left(\mathbf{r}, t^{\prime}\right)\right\rangle+\left\langle F_{0 s}^{(-)}\left(\mathbf{r}, t^{\prime}\right) J F_{0 s}^{(+)}(\mathbf{r}, t)\right\rangle\right\},
\end{aligned}
$$


where \langle\rangle denotes averaging by the Wigner density corresponding to the vacuum state. We have taken into account that $F_{0 s}^{(+)}$and $F_{0 i}^{(+)}$are uncorrelated. There are contributions of all terms of the expansion (35) of the field. Now, we are going to relate these contributions by using the preservation of commutation rules. If we consider the signal beam emerging from the crystal at different times $t$ and $t^{\prime}$, from Eqs. (24) and (5) we obtain

$$
\left[\hat{F}_{s}^{(+)}(\mathbf{r}, t), \hat{F}_{s}^{(-)}\left(\mathbf{r}, t^{\prime}\right)\right]=\left[\hat{F}_{0 s}^{(+)}(\mathbf{r}, t), \hat{F}_{0 s}^{(-)}\left(\mathbf{r}, t^{\prime}\right)\right],
$$

where $\hat{F}_{s}^{(+)}$is the field operator in the Heisenberg picture. Taking the expectation value in the vacuum for the latter expression and working in the Wigner representation, we obtain

$$
\begin{aligned}
\left\langle F_{0 s}^{(+)}(\mathbf{r}, t) J^{*} F_{0 s}^{(-)}\left(\mathbf{r}, t^{\prime}\right)\right\rangle & +\left\langle F_{0 s}^{(-)}\left(\mathbf{r}, t^{\prime}\right) J F_{0 s}^{(+)}(\mathbf{r}, t)\right\rangle \\
& =\left\langle G F_{0 i}^{(-)}(\mathbf{r}, t) G^{*} F_{0 i}^{(+)}\left(\mathbf{r}, t^{\prime}\right)\right\rangle .
\end{aligned}
$$

Therefore

$$
\begin{aligned}
& \left\langle F_{s}^{(+)}(\mathbf{r}, t) F_{s}^{(-)}\left(\mathbf{r}, t^{\prime}\right)\right\rangle-\left\langle F_{0 s}^{(+)}(\mathbf{r}, t) F_{0 s}^{(-)}\left(\mathbf{r}, t^{\prime}\right)\right\rangle \\
& \quad=2 g^{2}|V|^{2}\left\langle G F_{0 i}^{(-)}(\mathbf{r}, t) G^{*} F_{0 i}^{(+)}\left(\mathbf{r}, t^{\prime}\right)\right\rangle=g^{2}|V|^{2} \mu_{s}\left(t^{\prime}-t\right),
\end{aligned}
$$

where we have used that the processes involved are stationary and so the field correlations depend only on the time difference. $\mu_{s}(\tau)$ is a correlation function that we will not write explicitly, but that may be calculated if the function $f\left(\mathbf{k}, \mathbf{k}^{\prime}\right)$, introduced in the Hamiltonian (14), is known. It goes to zero when $\tau$ is greater than the correlation time of the signal $\tau_{s}$. Similarly,

$$
\begin{aligned}
& \left\langle F_{i}^{(+)}(\mathbf{r}, t) F_{i}^{(-)}\left(\mathbf{r}, t^{\prime}\right)\right\rangle-\left\langle F_{0 i}^{(+)}(\mathbf{r}, t) F_{0 i}^{(-)}\left(\mathbf{r}, t^{\prime}\right)\right\rangle \\
& \quad=g^{2}|V|^{2} \mu_{i}\left(t^{\prime}-t\right) .
\end{aligned}
$$

The following autocorrelations, and their complex conjugates, are zero:

$$
\left\langle F_{s}^{(+)}(\mathbf{r}, t) F_{s}^{(+)}\left(\mathbf{r}, t^{\prime}\right)\right\rangle=\left\langle F_{i}^{(+)}(\mathbf{r}, t) F_{i}^{(+)}\left(\mathbf{r}, t^{\prime}\right)\right\rangle=0 .
$$

\section{B. Cross correlations}

Also the cross correlation may be calculated. Taking the signal and idler fields at two points $(\mathbf{r}, t)$ and $\left(\mathbf{r}^{\prime}, t^{\prime}\right)$, respectively, we have

$$
\begin{aligned}
\left\langle F_{s}^{(+)}(\mathbf{r}, t) F_{i}^{(+)}\left(\mathbf{r}^{\prime}, t^{\prime}\right)\right\rangle= & \left\langle\left\{\left(1+g^{2}|V|^{2} J\right) F_{0 s}^{(+)}(\mathbf{r}, t)\right.\right. \\
& \left.+g V G F_{0 i}^{(-)}(\mathbf{r}, t)\right\} \\
& \times\left\{\left(1+g^{2}|V|^{2} J\right) F_{0 i}^{(+)}\left(\mathbf{r}^{\prime}, t^{\prime}\right)\right. \\
& \left.\left.+g V G F_{0 s}^{(-)}\left(\mathbf{r}^{\prime}, t^{\prime}\right)\right\}\right\rangle \\
= & g V\left[\left\langle F_{0 s}^{(+)}(\mathbf{r}, t) G F_{0 s}^{(-)}\left(\mathbf{r}^{\prime}, t^{\prime}\right)\right\rangle\right. \\
& \left.+\left\langle F_{0 i}^{(+)}\left(\mathbf{r}^{\prime}, t^{\prime}\right) G F_{0 i}^{(-)}(\mathbf{r}, t)\right\rangle\right] .
\end{aligned}
$$

Now we are going to apply again the conservation of commutation rules, but by considering the signal and idler field operators at different times. By taking into account the same considerations as above, we have

$$
\begin{gathered}
{\left[\hat{F}_{s}^{(+)}(\mathbf{r}, t), \hat{F}_{i}^{(+)}\left(\mathbf{r}^{\prime}, t^{\prime}\right)\right]=\left[\hat{F}_{0 s}^{(+)}(\mathbf{r}, t), \hat{F}_{0 i}^{(+)}\left(\mathbf{r}^{\prime}, t^{\prime}\right)\right]=0 \rightarrow} \\
\left\langle F_{0 s}^{(+)}(\mathbf{r}, t) G F_{0 s}^{(-)}\left(\mathbf{r}^{\prime}, t^{\prime}\right)\right\rangle=\left\langle F_{0 i}^{(+)}\left(\mathbf{r}^{\prime}, t^{\prime}\right) G F_{0 i}^{(-)}(\mathbf{r}, t)\right\rangle .
\end{gathered}
$$

The second conmutator is zero because it contains only destruction operators. From Eqs. (43) and (44), it follows that

$$
\left\langle F_{s}^{(+)}(\mathbf{0}, t) F_{i}^{(+)}\left(\mathbf{0}, t^{\prime}\right)\right\rangle=g V \nu\left(t^{\prime}-t\right) .
$$

The corss-correlation for $\mathbf{r}$ and $\mathbf{r}^{\prime}$ different from $\mathbf{0}$ may be obtained using Eq. (34). The function $\nu(\tau)$ defines a coherence time $\tau_{s i}$ between signal and idler. By a similar argument, it can be proved that

$$
\left\langle F_{s}^{(+)}(\mathbf{r}, t) F_{i}^{(-)}\left(\mathbf{r}^{\prime}, t^{\prime}\right)\right\rangle=\left\langle F_{s}^{(-)}(\mathbf{r}, t) F_{i}^{(+)}\left(\mathbf{r}^{\prime}, t^{\prime}\right)\right\rangle=0 .
$$

\section{DETECTION PROBABILITIES}

In the Hilbert-space formalism, the usual theory of detection (by photon absorption) is based on normal ordering. Single and joint detection rates are given by

$$
\begin{gathered}
P_{a}\left(\mathbf{r}_{a}, t\right)=K\left\langle 0\left|\hat{E}^{(-)}\left(\mathbf{r}_{a}, t\right) \hat{E}^{(+)}\left(\mathbf{r}_{a}, t\right)\right| 0\right\rangle, \\
P_{a b}\left(\mathbf{r}_{a}, t ; \mathbf{r}_{b}, t^{\prime}\right)= \\
K^{\prime}\langle 0| \hat{E}^{(-)}\left(\mathbf{r}_{a}, t\right) \hat{E}^{(-)}\left(\mathbf{r}_{b}, t^{\prime}\right) \\
\\
\times \hat{E}^{(+)}\left(\mathbf{r}_{b}, t^{\prime}\right) \hat{E}^{(+)}\left(\mathbf{r}_{a}, t\right)|0\rangle
\end{gathered}
$$

in the Heisenberg picture, where $K$ and $K^{\prime}$ are two constants related to the efficiency of the detectors.

We now formulate the quantum theory of detection in the Wigner representation. The corresponding probabilities are now

$$
\begin{gathered}
P_{a}\left(\mathbf{r}_{a}, t\right)=K\left\langle I\left(\mathbf{r}_{a}, t\right)-I_{0}\left(\mathbf{r}_{a}\right)\right\rangle, \\
P_{a b}\left(\mathbf{r}_{a}, t ; \mathbf{r}_{b}, t^{\prime}\right)=K^{\prime}\left\langle\left\{I\left(\mathbf{r}_{a}, t\right)-I_{0}\left(\mathbf{r}_{a}\right)\right\}\left\{I\left(\mathbf{r}_{b}, t^{\prime}\right)-I_{0}\left(\mathbf{r}_{b}\right)\right\}\right\rangle,
\end{gathered}
$$

where $I(\mathbf{r}, t)=\left|E^{(+)}(\mathbf{r}, t)\right|^{2}$ and $I_{0}(\mathbf{r})=\left\langle\left|E^{(+)}(\mathbf{r},-\Delta t)\right|^{2}\right\rangle$, $E^{(+)}(\mathbf{r}, t)$ being defined by Eq. (6).

We prove Eq. (49) as follows: 


$$
\begin{aligned}
\left\langle 0\left|\hat{E}^{(-)}\left(\mathbf{r}_{a}, t\right) \hat{E}^{(+)}\left(\mathbf{r}_{a}, t\right)\right| 0\right\rangle= & \frac{1}{2}\left\langle 0\left|\hat{E}^{(+)}\left(\mathbf{r}_{a}, t\right) \hat{E}^{(-)}\left(\mathbf{r}_{a}, t\right)+\hat{E}^{(-)}\left(\mathbf{r}_{a}, t\right) \hat{E}^{(+)}\left(\mathbf{r}_{a}, t\right)\right| 0\right\rangle \\
& -\frac{1}{2}\left\langle 0\left|\hat{E}^{(+)}\left(\mathbf{r}_{a}, t\right) \hat{E}^{(-)}\left(\mathbf{r}_{a}, t\right)-\hat{E}^{(-)}\left(\mathbf{r}_{a}, t\right) \hat{E}^{(+)}\left(\mathbf{r}_{a}, t\right)\right| 0\right\rangle \\
= & \left\langle 0\left|S\left\{\hat{E}^{(+)}\left(\mathbf{r}_{a}, t\right) \hat{E}^{(-)}\left(\mathbf{r}_{a}, t\right)\right\}\right| 0\right\rangle-\frac{1}{2}\langle 0| \hat{U}^{\dagger}(t+\Delta t) \\
& \times\left[\hat{E}^{(+)}\left(\mathbf{r}_{a},-\Delta t\right), \hat{E}^{(-)}\left(\mathbf{r}_{a},-\Delta t\right)\right] \hat{U}(t+\Delta t)|0\rangle \\
= & \left\langle E^{(+)}\left(\mathbf{r}_{a}, t\right) E^{(-)}\left(\mathbf{r}_{a}, t\right)\right\rangle-\frac{1}{2}\left\langle 0\left|\left[\hat{E}^{(+)}\left(\mathbf{r}_{a},-\Delta t\right), \hat{E}^{(-)}\left(\mathbf{r}_{a},-\Delta t\right)\right]\right| 0\right\rangle \\
= & \left\langle I\left(\mathbf{r}_{a}, t\right)\right\rangle-\left\langle 0\left|S\left\{\hat{E}^{(+)}\left(\mathbf{r}_{a},-\Delta t\right) \hat{E}^{(-)}\left(\mathbf{r}_{a},-\Delta t\right)\right\}\right| 0\right\rangle \\
= & \left\langle I\left(\mathbf{r}_{a}, t\right)\right\rangle-\left\langle I\left(\mathbf{r}_{a}, 0\right)\right\rangle=\left\langle I\left(\mathbf{r}_{a}, t\right)-I_{0}\left(\mathbf{r}_{a}\right)\right\rangle,
\end{aligned}
$$

where $\hat{U}(t+\Delta t)$ is the evolution operator. The third equality follows because the commutator of $\hat{E}^{(+)}\left(\mathbf{r}_{a},-\Delta t\right)$ and $\hat{E}^{(-)}\left(\mathbf{r}_{a},-\Delta t\right)$ is a $c$ number (that does not change during the evolution). The fourth follows because the vacuum expectation of a normally ordered product vanishes, and this allows us to replace the commutator by the anticommutator and then write it in terms of symmetrical ordering.

Equation (50) is a little more involved. After some tedious algebra it can be proved that

$$
\begin{aligned}
& \left\langle 0\left|\hat{E}^{(-)}\left(\mathbf{r}_{a}, t\right) \hat{E}^{(-)}\left(\mathbf{r}_{b}, t^{\prime}\right) \hat{E}^{(+)}\left(\mathbf{r}_{b}, t^{\prime}\right) \hat{E}^{(+)}\left(\mathbf{r}_{a}, t\right)\right| 0\right\rangle=\langle 0| S\left\{\left(\hat{E}^{(+)}\left(\mathbf{r}_{a}, t\right) \hat{E}^{(-)}\left(\mathbf{r}_{a}, t\right)-\frac{1}{2}\left[\hat{E}^{(+)}\left(\mathbf{r}_{a}, t\right), \hat{E}^{(-)}\left(\mathbf{r}_{a}, t\right)\right]\right)\right. \\
& \left.\times\left(\hat{E}^{(+)}\left(\mathbf{r}_{b}, t^{\prime}\right) \hat{E}^{(-)}\left(\mathbf{r}_{b}, t^{\prime}\right)-\frac{1}{2}\left[\hat{E}^{(+)}\left(\mathbf{r}_{b}, t^{\prime}\right), \hat{E}^{(-)}\left(\mathbf{r}_{b}, t^{\prime}\right)\right]\right)\right\}|0\rangle \\
& +\frac{1}{2}\langle 0|\left[\hat{E}^{(+)}\left(\mathbf{r}_{a}, t\right), \hat{E}^{(+)}\left(\mathbf{r}_{b}, t^{\prime}\right)\right]\left\{S\left(\hat{E}^{(-)}\left(\mathbf{r}_{a}, t\right) \hat{E}^{(-)}\left(\mathbf{r}_{b}, t^{\prime}\right)\right)\right. \\
& \left.-\left[\hat{E}^{(-)}\left(\mathbf{r}_{a}, t\right), \hat{E}^{(-)}\left(\mathbf{r}_{b}, t^{\prime}\right)\right]\right\}|0\rangle+\frac{1}{2}\langle|\left[\hat{E}^{(-)}\left(\mathbf{r}_{b}, t^{\prime}\right), \hat{E}^{(+)}\left(\mathbf{r}_{a}, t\right)\right] \\
& \times\left\{S\left(\hat{E}^{(+)}\left(\mathbf{r}_{b}, t^{\prime}\right) \hat{E}^{(-)}\left(\mathbf{r}_{a}, t\right)\right)-\left[\hat{E}^{(+)}\left(\mathbf{r}_{b}, t^{\prime}\right), \hat{E}^{(-)}\left(\mathbf{r}_{a}, t\right)\right]\right\}|0\rangle \\
& +\frac{1}{2}\langle|\left[\hat{E}^{(-)}\left(\mathbf{r}_{a}, t\right), \hat{E}^{(+)}\left(\mathbf{r}_{b}, t^{\prime}\right)\right]\left\{S\left(\hat{E}^{(+)}\left(\mathbf{r}_{a}, t\right) \hat{E}^{(-)}\left(\mathbf{r}_{b}, t^{\prime}\right)\right)\right. \\
& \left.-\left[\hat{E}^{(+)}\left(\mathbf{r}_{a}, t\right), \hat{E}^{(-)}\left(\mathbf{r}_{b}, t^{\prime}\right)\right]\right\}|0\rangle+\frac{1}{2}\langle 0|\left[\hat{E}^{(-)}\left(\mathbf{r}_{b}, t^{\prime}\right), \hat{E}^{(-)}\left(\mathbf{r}_{a}, t\right)\right] \\
& \times\left\{S\left(\hat{E}^{(+)}\left(\mathbf{r}_{b}, t^{\prime}\right) \hat{E}^{(+)}\left(\mathbf{r}_{a}, t\right)\right)-\left[\hat{E}^{(+)}\left(\mathbf{r}_{b}, t^{\prime}\right), \hat{E}^{(+)}\left(\mathbf{r}_{a}, t\right)\right]\right\}|0\rangle,
\end{aligned}
$$


which is similar to Wick's theorem [[9], but in this case it establishes the relation between normal and symmetrical ordering instead of normal and time ordering.

By the same argument as that used in formula (51), for transforming the anticommutator into the intensity of the vacuum field, the two commutators can be written as

$$
\begin{gathered}
\frac{1}{2}\left[\hat{E}^{(+)}\left(\mathbf{r}_{a}, t\right), \hat{E}^{(-)}\left(\mathbf{r}_{a}, t\right)\right]=\left\langle\left|E^{(+)}\left(\mathbf{r}_{a},-\Delta t\right)\right|^{2}\right\rangle=I_{0}\left(\mathbf{r}_{a}\right), \\
\frac{1}{2}\left[\hat{E}^{(+)}\left(\mathbf{r}_{b}, t^{\prime}\right), \hat{E}^{(-)}\left(\mathbf{r}_{b}, t^{\prime}\right)\right]=\left\langle\left|E^{(+)}\left(\mathbf{r}_{b},-\Delta t\right)\right|^{2}\right\rangle=I_{0}\left(\mathbf{r}_{b}\right) .
\end{gathered}
$$

On the other hand, the rest of commutators are zero in PDC experiments because they involve different modes of the radiation field. Then the result (50) follows by taking into account these two facts in Eq. (52) and writing the vacuum expectation values of the remaining symmetrical operators as the corresponding averages with the Wigner density.

For the purpose of applying Eq. (50) to the experiments, it is convenient to write it in a more compact form. We use the fact, proved in Sec. IV, that the Wigner field amplitudes are Gaussian processes. For four Gaussian random variables $A$, $B, C$, and $D$, the well-known property

$$
\langle A B C D\rangle=\langle A B\rangle\langle C D\rangle+\langle A C\rangle\langle B D\rangle+\langle A D\rangle\langle B C\rangle
$$

allows us to write the coincidence probability as

$$
\begin{aligned}
P_{a b}\left(\mathbf{r}_{a}, t ; \mathbf{r}_{b}, t^{\prime}\right)= & K^{\prime}\left\langle\left\{I\left(\mathbf{r}_{a}, t\right)-I_{0}\left(\mathbf{r}_{a}\right)\right\}\left\{I\left(\mathbf{r}_{b}, t^{\prime}\right)-I_{0}\left(\mathbf{r}_{b}\right)\right\}\right\rangle \\
= & P_{a}\left(\mathbf{r}_{a}, t\right) P_{b}\left(\mathbf{r}_{b}, t^{\prime}\right) \\
& +K^{\prime}\left|\left\langle E^{(+)}\left(\mathbf{r}_{a}, t\right) E^{(-)}\left(\mathbf{r}_{b}, t^{\prime}\right)\right\rangle\right|^{2} \\
& +K^{\prime}\left|\left\langle E^{(+)}\left(\mathbf{r}_{a}, t\right) E^{(+)}\left(\mathbf{r}_{b}, t^{\prime}\right)\right\rangle\right|^{2} .
\end{aligned}
$$

On the other hand, from Sec. IV it can be easily seen that the first two terms are fourth-order in $g$, while the last term is second-order in $g$. This means that we may discard the first two terms and finally obtain

$$
P_{a b}\left(\mathbf{r}_{a}, t ; \mathbf{r}_{b}, t^{\prime}\right)=K^{\prime}\left|\left\langle E^{(+)}\left(\mathbf{r}_{a}, t\right) E^{(+)}\left(\mathbf{r}_{b}, t^{\prime}\right)\right\rangle\right|^{2} .
$$

In actual experiments there is always a detection window $w$ and we should perform the time integral of Eq. (56) within this window. We have

$$
\begin{aligned}
P_{a b}= & \frac{\eta^{2}}{\hbar^{2} \omega_{a} \omega_{b}} \int_{0}^{w} d \tau \int_{0}^{w} d \tau^{\prime} \mid\left\langle E^{(+)}\left(\mathbf{r}_{a}, t+\tau\right)\right. \\
& \left.\times E^{(+)}\left(\mathbf{r}_{b}, t+\tau^{\prime}\right)\right\rangle\left.\right|^{2} .
\end{aligned}
$$

We have assumed that radiation modes where the amplitude differs significantly from the zero-point value are concentrated in narrow bands around $\omega_{a}$ and $\omega_{b}$, respectively. For simplicity we have assumed also that the two windows are identical and that both detectors have the same efficiency $\eta$.

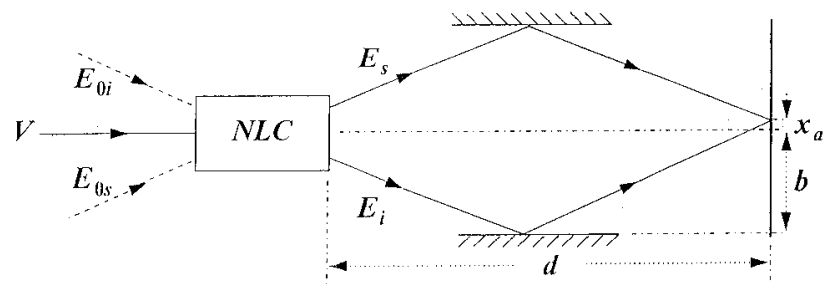

FIG. 2. Experiment of interference on a screen by Ghosh and Mandel.

\section{EXPERIMENT ON THE INTERFERENCE OF SIGNAL AND IDLER PHOTONS}

The coherence properties of PDC photon pairs were investigated by Ghosh et al. [10]. They directed degenerate signal and idler beams towards a screen by means of two mirrors (see Fig. 2), where "degenerate" means that the directions of the signal and idler beams are so chosen that $\omega_{s}=\omega_{i}=\omega_{0} / 2$. If one detector is put on the screen, no change is observed in the counting rate when the detector changes position. This shows that there is no second-order interference between the two beams. In contrast, when two detectors are put on the screen, the coincidence counting rate is observed to depend on the relative position of the detectors. This shows a fourth-order, or intensity-intensity, interference.

The explanation of these results, in the Wigner representation, is straightforward. In the scalar approximation (valid because the angle between signal and idler beams is small) the field at a point $\mathbf{r}_{a}$ on the screen (see Fig. 2) is, using Eq. (34),

$$
\begin{aligned}
F^{(+)}\left(\mathbf{r}_{a}, t\right)= & F_{s}^{(+)}\left(\mathbf{r}_{a}, t\right)+F_{i}^{(+)}\left(\mathbf{r}_{a}, t\right) \\
= & F_{s}^{(+)}\left(\mathbf{0}, t-r_{s} / c\right) e^{i \omega_{0} r_{s} / 2 c} \\
& +F_{i}^{(+)}\left(\mathbf{0}, t-r_{i} / c\right) e^{i \omega_{0} r_{i} / 2 c} .
\end{aligned}
$$

The vector $\mathbf{r}_{a}$ has components $\left(x_{a}, y_{a}, d\right), d$ being the distance from the center of the crystal to the screen. The optical path lengths of the signal $r_{s}$ and idler $r_{i}$ beams from the center of the crystal to the detectors are

$$
r_{s}=\sqrt{\left(2 b-x_{a}\right)^{2}+y_{a}^{2}+d^{2}}, \quad r_{i}=\sqrt{\left(2 b+x_{a}\right)^{2}+y_{a}^{2}+d^{2}},
$$

where $b$ is the distance from the axis of the pumping to the mirrors.

For the calculation of the single detection probability, using Eq. (49), we need the average

$$
\begin{aligned}
\left\langle I\left(\mathbf{r}_{a}, t\right)-I_{0}\left(\mathbf{r}_{a}\right)\right\rangle= & \left\langle\left|F^{(+)}\left(\mathbf{r}_{a}, t\right)\right|^{2}\right\rangle-\left\langle\left|F^{(+)}\left(\mathbf{r}_{a}, 0\right)\right|^{2}\right\rangle \\
= & \left\langle\left|F_{s}^{(+)}\left(\mathbf{r}_{a}, t\right)\right|^{2}\right\rangle-\left\langle\left|F_{s}^{(+)}\left(\mathbf{r}_{a}, 0\right)\right|^{2}\right\rangle \\
& +\left\langle\left|F_{i}^{(+)}\left(\mathbf{r}_{a}, t\right)\right|^{2}\right\rangle-\left\langle\left|F_{i}^{(+)}\left(\mathbf{r}_{a}, 0\right)\right|^{2}\right\rangle \\
& +2 \operatorname{Re}\left[\left\langleF_{s}^{(+)}\left(\mathbf{0}, t-r_{s} / c\right)\right.\right. \\
& \left.\left.\times F_{i}^{(-)}\left(\mathbf{0}, t-r_{i} / c\right)\right\rangle e^{i \omega_{0}\left(r_{s}-r_{i}\right) / 2 c}\right] .
\end{aligned}
$$


The last term is zero because $F_{s}^{(+)}$and $F_{i}^{(-)}$are uncorrelated [see Eq. (46)]. The other four terms give no dependence on $\mathbf{r}_{a}$, that is, we get no interference between signal and idler.
The coincidence probability in two detectors placed at $\mathbf{r}_{a}$ and $\mathbf{r}_{b}$ is given by Eq. (56). The amplitude $F^{(+)}\left(\mathbf{r}_{a}, t\right)$ was given in Eq. (58) and a similar expression is valid for $F^{(+)}\left(\mathbf{r}_{b}, t^{\prime}\right)$. Then, we have

$$
\begin{aligned}
P_{a b}\left(\mathbf{r}_{a}, t+\tau ; \mathbf{r}_{b}, t+\tau^{\prime}\right)= & K^{\prime} \mid\left\langle F_{s}^{(+)}\left(\mathbf{r}_{a}, t+\tau\right) F_{s}^{(+)}\left(\mathbf{r}_{b}, t+\tau^{\prime}\right)+F_{i}^{(+)}\left(\mathbf{r}_{a}, t+\tau\right) F_{i}^{(+)}\left(\mathbf{r}_{b}, t+\tau^{\prime}\right)\right. \\
& \left.+F_{s}^{(+)}\left(\mathbf{r}_{a}, t+\tau\right) F_{i}^{(+)}\left(\mathbf{r}_{b}, t+\tau^{\prime}\right)+F_{i}^{(+)}\left(\mathbf{r}_{a}, t+\tau\right) F_{s}^{(+)}\left(\mathbf{r}_{b}, t+\tau^{\prime}\right)\right\rangle\left.\right|^{2} \\
= & \left|\left\langle F_{s}^{(+)}\left(\mathbf{r}_{a}, t+\tau\right) F_{i}^{(+)}\left(\mathbf{r}_{b}, t+\tau^{\prime}\right)+F_{i}^{(+)}\left(\mathbf{r}_{a}, t+\tau\right) F_{s}^{(+)}\left(\mathbf{r}_{b}, t+\tau^{\prime}\right)\right\rangle\right|^{2} \\
= & \mid\left\langle F_{s}^{(+)}\left(\mathbf{0}, t+\tau-r_{s} / c\right) F_{i}^{(+)}\left(\mathbf{0}, t+\tau^{\prime}-r_{i}^{\prime} / c\right)\right\rangle e^{i \omega_{0}\left(r_{s}+r_{i}^{\prime}\right) / 2 c} \\
& +\left.\left\langle F_{i}^{(+)}\left(\mathbf{0}, t+\tau-r_{i} / c\right) F_{s}^{(+)}\left(\mathbf{0}, t+\tau^{\prime}-r_{s}^{\prime} / c\right)\right\rangle e^{i \omega_{0}\left(r_{s}^{\prime}+r_{i}\right) / 2 c}\right|^{2},
\end{aligned}
$$

where we have used Eq. (46) in the first equation and Eq. (34) in the second.

Now, expanding $r_{s}$ and $r_{i}\left(r_{s}^{\prime}\right.$ and $\left.r_{i}^{\prime}\right)$ to first order in $x_{a}\left(x_{b}\right)$ and using Eq. (57) we get

$$
\begin{aligned}
P_{a b}\left(x_{a} ; x_{b}\right) \approx & \left(\frac{2 \eta}{\hbar \omega_{0}}\right)^{2} g^{2}|V|^{2} \int_{0}^{w} d \tau \int_{0}^{w} d \tau^{\prime} \\
& \times\left(\left|\nu\left[\tau^{\prime}-\tau-b\left(x_{a}+x_{b}\right) / c d\right]\right|^{2}\right. \\
& +\left|\nu\left[\tau-\tau^{\prime}-b\left(x_{a}+x_{b}\right) / c d\right]\right|^{2} \\
& +2 \operatorname{Re}\left\{\nu\left[\tau^{\prime}-\tau-b\left(x_{a}+x_{b}\right) / c d\right]\right. \\
& \times \nu^{*}\left[\tau-\tau^{\prime}-b\left(x_{a}+x_{b}\right) / c d\right] \\
& \left.\left.\times e^{i \omega_{0} b\left(x_{b}-x_{a}\right) / c d}\right\}\right),
\end{aligned}
$$

which shows a cosine dependence on $\omega_{0} b\left(x_{b}-x_{a}\right) / c d$ $=b\left(x_{b}-x_{a}\right) / \lambda_{0} d$. It is easy to see that if the detection window $w$ is much bigger than the correlation time of $\nu(\tau)$ and this is bigger than the quantity $b\left(x_{a}+x_{b}\right) / c d$, then this quantity may be neglected in the argument of the function $\nu$. In these conditions, because $|\nu(\tau)|$ is an even function of $\tau$ (see [10]), the visibility of the interference pattern given by Eq. (62) becomes close to $100 \%$.

\section{THE EXPERIMENT OF RARITY AND TAPSTER}

In 1990 Rarity and Tapster [11] performed an experiment to test Bell's inequality using phase and momentum of photon pairs (instead of polarization as in previous experiments). The experiment consisted of selecting two signal beams of the same color (frequency $\omega_{s}$ ) and two idler beams also of

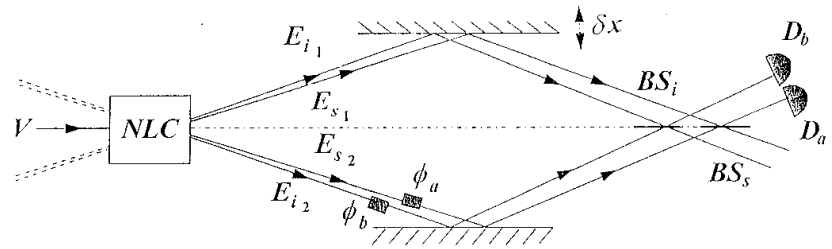

FIG. 3. Experiment of Rarity and Tapster. the same color (frequency $\omega_{i}$, different from $\omega_{s}$ ). One of the signal beams and one of the idler beams go to a mirror put above the pumping beam axis and the other two at another mirror put below (see Fig. 3). The two signals are recombined at one beam splitter and the two idlers at another (in practice, the authors used two differents points of the same beam splitter). Two detectors were put in appropriate places to detect the signal and the idler, respectively. Two controllable phase shifters were introduced in the lower beams, so that the phase of the signal was increased by $\phi_{a}$ and that of the idler by $\phi_{b}$. The result of the experiment was that the coincidence detection probability had a cosine variation with $\phi_{a}-\phi_{b}$ with a visibility close to $100 \%$. In the actual experiment the signal and idler detectors were placed near each other, but, in principle, it is possible to send the signal and idler beams to two distant regions of space by using appropriate mirrors placed near the crystal. For this reason the experiment has been interpreted as a violation of a Bell inequality [11].

In the Wigner representation the field amplitudes arriving at the signal and idler detectors (placed at $\mathbf{r}_{a}$ and $\mathbf{r}_{b}$, respectively) will be, using Eq. (12),

$$
\begin{gathered}
F_{s}^{(+)}\left(\mathbf{r}_{a}, t\right)=i R_{s} F_{s_{1}}^{(+)}\left(\mathbf{r}_{a}, t\right)+T_{s} F_{s_{2}}^{(+)}\left(\mathbf{r}_{a}, t\right), \\
F_{i}^{(+)}\left(\mathbf{r}_{b}, t^{\prime}\right)=T_{i} F_{i_{1}}^{(+)}\left(\mathbf{r}_{b}, t^{\prime}\right)+i R_{i} F_{i_{2}}^{(+)}\left(\mathbf{r}_{b}, t^{\prime}\right),
\end{gathered}
$$

where we have labeled 1 (2) the upper (lower) beams. $T_{S}$ and $R_{s}\left(T_{i}\right.$ and $\left.R_{i}\right)$ are the transmission and reflection coefficients of the signal (idler) in the beam splitter (see Fig. 3). Now we take Eq. (34) into account and write

$$
\begin{gathered}
F_{s_{1}}^{(+)}\left(\mathbf{r}_{a}, t\right)=e^{i \omega_{s}\left(r_{a}+\delta x\right) / c} F_{s_{1}}^{(+)}\left(\mathbf{0}, t-r_{a} / c+\delta x / c\right), \\
F_{s_{2}}^{(+)}\left(\mathbf{r}_{a}, t\right)=e^{i \omega_{s} r_{a} / c+i \phi_{a} F_{s_{2}}^{(+)}\left(\mathbf{0}, t-r_{a} / c+\phi_{a} / \omega_{s}\right),} \\
F_{i_{1}}^{(+)}\left(\mathbf{r}_{b}, t^{\prime}\right)=e^{i \omega_{i}\left(r_{b}+\delta x\right) / c} F_{i_{1}}^{(+)}\left(\mathbf{0}, t^{\prime}-r_{b} / c+\delta x / c\right), \\
F_{i_{2}}^{(+)}\left(\mathbf{r}_{b}, t^{\prime}\right)=e^{i \omega_{i} r_{b} / c+i \phi_{b}} F_{i_{2}}^{(+)}\left(\mathbf{0}, t^{\prime}-r_{b} / c+\phi_{b} / \omega_{i}\right) .
\end{gathered}
$$


We have labeled $r_{a}\left(r_{b}\right)$ the path length of the lower signal (idler) beam from the center of the crystal to the detectors. In the actual experiment both upper paths are modified by $\delta x$ because the upper and lower mirrors are not at exactly the same distance from the pumping beam axis. However, in our calculation we shall set $\delta x=0$ and $r_{a}=r_{b} \equiv r$ for the sake of simplicity.

The coincidence detection probability is given by Eq. (57). We need the cross correlation

$$
\begin{aligned}
& \left|\left\langle F_{s}^{(+)}\left(\mathbf{r}_{a}, t+\tau\right) F_{i}^{(+)}\left(\mathbf{r}_{b}, t^{\prime}+\tau^{\prime}\right)\right\rangle\right| \\
& =\mid R_{s} T_{i} e^{i \phi_{b}}\left\langle F_{s_{1}}^{(+)}(\mathbf{0}, t+\tau-r / c)\right. \\
& \left.\times F_{i_{2}}^{(+)}\left(\mathbf{0}, t^{\prime}+\tau^{\prime}-r / c-\phi_{b} / \omega_{i}\right)\right\rangle \\
& +R_{i} T_{s} e^{i \phi_{a}\left\langle F_{s_{2}}^{(+)}\right.}\left(\mathbf{0}, t+\tau-r / c-\phi_{a} / \omega_{s}\right) \\
& \left.\times F_{i_{1}}^{(+)}\left(\mathbf{0}, t^{\prime}+\tau^{\prime}-r / c\right)\right\rangle \mid \\
& =\mid R_{s} T_{i} e^{i \phi_{b}} \nu\left(\tau^{\prime}-\tau-\phi_{b} / \omega_{i}\right) \\
& +R_{i} T_{s} e^{i \phi_{a}} \nu\left(\tau^{\prime}-\tau-\phi_{a} / \omega_{s}\right) \mid,
\end{aligned}
$$

where in the first equation we have taken into account that the signal field $F_{s_{1}}^{(+)}$is correlated with the idler field $F_{i_{2}}^{(+)}$ and also $F_{s_{2}}^{(+)}$is correlated with $F_{i_{1}}^{(+)}$, but $F_{s_{j}}^{(+)}$is uncorrelated with $F_{i_{j}}^{(+)}(j=1$ or 2$)$, these pairs not fulfilling the matching conditions (25). The second equality of Eq. (65) follows from Eq. (45). If we take into account that the time intervals $\phi_{a} / \omega_{s}$ and $\phi_{b} / \omega_{i}$ are small in comparison to the coherence time of signal and idler, given by the function $\nu(\tau)$, we obtain

$$
\begin{aligned}
P_{a b}\left(\phi_{a}, \phi_{b}\right) \approx & \frac{\eta^{2}}{\hbar^{2} \omega_{s} \omega_{i}} g^{2}|V|^{2} \int_{0}^{w} d \tau \int_{0}^{w} d \tau^{\prime}\left|\nu\left(\tau^{\prime}-\tau\right)\right|^{2} \\
& \times\left\{\left(R_{i} T_{s}\right)^{2}+\left(R_{s} T_{i}\right)^{2}+2 R_{s} T_{s} R_{i} T_{i}\right. \\
& \left.\times \cos \left(\phi_{a}-\phi_{b}\right)\right\} .
\end{aligned}
$$

We get a fourth-order interference with a visibility close to $100 \%$ provided that the reflection and transmission in the beam splitter fulfill $R_{i} / T_{i}=R_{s} / T_{s}$. A similar expression is obtained if both detectors are placed below the pumping beam axis. If one of the detectors is placed above and the other below we obtain

$$
\begin{aligned}
P_{a b}\left(\phi_{a}, \phi_{b}\right) \approx & \frac{\eta^{2}}{\hbar^{2} \omega_{s} \omega_{i}} g^{2}|V|^{2} \int_{0}^{w} d \tau \int_{0}^{w} d \tau^{\prime}\left|\nu\left(\tau^{\prime}-\tau\right)\right|^{2} \\
& \times\left\{\left(T_{s} T_{i}\right)^{2}+\left(R_{s} R_{i}\right)^{2}\right. \\
& \left.+2 R_{s} T_{s} R_{i} T_{i} \cos \left(\phi_{a}-\phi_{b}\right)\right\}
\end{aligned}
$$

In this case the $100 \%$ visibility requires $T_{s} T_{i}=R_{s} R_{i}$. If we want high visibility in both Eqs. (66) and (67), we need $T_{s}=T_{i}=R_{s}=R_{i}=1 / \sqrt{2}$.

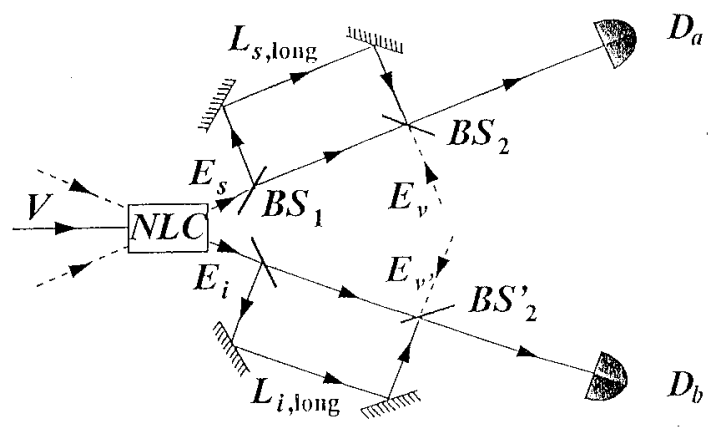

FIG. 4. Experiment of Franson.

\section{THE EXPERIMENTS OF FRANSON}

In 1989 Franson proposed an experiment in order to test " a Bell's inequality for energy and time" [12]. The experiment consists of the following (see Fig. 4). A signal and an idler beam are passed each through a Mach-Zehnder interferometer, that is, an arrangement of two beam splitters (BS) and two mirrors. The signal beam is divided at the first $\mathrm{BS}_{1}$ and two outgoing beams travel by different routes, one long and the other one short, until a second $\mathrm{BS}_{2}$ where they are recombined. One of the outgoing beams at this $\mathrm{BS}_{2}$ goes to a detector. A similar thing happens with the idler beam. We shall label $\Delta L_{s}\left(\Delta L_{i}\right)$ the length difference between the long and the short route of the signal (idler) beam. In the experiment it is observed that the single detection rates do not depend on $\Delta L_{s}$ or $\Delta L_{i}$, but the coincidence detection rate exhibits a cosine dependence on $\left(\omega_{s} \Delta L_{s}+\omega_{i} \Delta L_{i}\right) / c$, which shows a fourth-order interference. In the past few years several groups have performed experiments of that type, the most recent one by Tapster et al. [13] (references to previous experiments may be seen in that paper).

In order to compute the joint detection probability, we must write the fields at the detectors as functions of the outcoming fields from the crystal. Without a loss of generality we shall neglect the distances from the crystal to MachZehnder interferometers in order to simplify notation (see Fig. 4).

If we call $\mathbf{r}_{a}\left(\mathbf{r}_{b}\right)$ the position of detector $D_{a}\left(D_{b}\right), \mathbf{r}_{2}$ $\left(\mathbf{r}_{2}^{\prime}\right)$ the position of $\mathrm{BS}_{2}\left(\mathrm{BS}_{2}^{\prime}\right), L_{s}$, long $\left(L_{i, \text { long }}\right)$ the length of the long arm of the interferometer, and $L_{s \text {, short }}\left(L_{i \text {, short }}\right)$ the length of the short arm of the interferometer for the signal (idler) beam, then

$$
\begin{aligned}
F_{s}^{(+)}\left(\mathbf{r}_{a}, t\right)= & \frac{1}{2}\left\{\left[F_{s}^{(+)}\left(\mathbf{0}, t-\frac{\left|\mathbf{r}_{a}-\mathbf{r}_{2}\right|}{c}-\frac{L_{s, \text { long }}}{c}\right)\right.\right. \\
& \left.-i F_{v}^{(+)}\left(\mathbf{0}, t-\frac{\left|\mathbf{r}_{a}-\mathbf{r}_{2}\right|}{c}-\frac{\left.L_{s, \text { long }}\right)}{c}\right)\right] e^{i \omega_{s}\left(L_{s, \text { long }} / c\right)} \\
& +\left[F_{s}^{(+)}\left(\mathbf{0}, t-\frac{\left|\mathbf{r}_{a}-\mathbf{r}_{2}\right|}{c}-\frac{L_{s, \text { short }}}{c}\right)\right. \\
& \left.+i F_{v}^{(+)}\left(\mathbf{0}, t-\frac{\left|\mathbf{r}_{a}-\mathbf{r}_{2}\right|}{c}-\frac{L_{s, \text { short }}}{c}\right)\right] \\
& \left.\times e^{i \omega_{s}\left(L_{s, \text { short }} / c\right)}\right\} e^{i \omega_{s}\left(\left|\mathbf{r}_{a}-\mathbf{r}_{2}\right| / c\right)},
\end{aligned}
$$




$$
\begin{aligned}
F_{i}^{(+)}\left(\mathbf{r}_{b}, t^{\prime}\right)= & \frac{1}{2}\left\{\left[F_{i}^{(+)}\left(\mathbf{0}, t^{\prime}-\frac{\left|\mathbf{r}_{b}-\mathbf{r}_{2}^{\prime}\right|}{c}-\frac{L_{i, \text { long }}}{c}\right)\right.\right. \\
& \left.-i F_{v^{\prime}}^{(+)}\left(\mathbf{0}, t^{\prime}-\frac{\left|\mathbf{r}_{b}-\mathbf{r}_{2}^{\prime}\right|}{c}-\frac{\left.L_{i, \text { long }}\right)}{c}\right)\right] \\
& \times e^{i \omega_{i}\left(L_{i, \text { long }} / c\right)} \\
& +\left[F_{i}^{(+)}\left(\mathbf{0}, t^{\prime}-\frac{\left|\mathbf{r}_{b}-\mathbf{r}_{2}^{\prime}\right|}{c}-\frac{L_{i, \text { short }}}{c}\right)\right. \\
& \left.+i F_{v^{\prime}}^{(+)}\left(\mathbf{0}, t^{\prime}-\frac{\left|\mathbf{r}_{b}-\mathbf{r}_{2}^{\prime}\right|}{c}-\frac{L_{i, \text { short }}}{c}\right)\right] \\
& \left.\times e^{i \omega_{i}\left(L_{i, \text { short }} / c\right)}\right\} e^{i \omega_{i}\left(\left|\mathbf{r}_{b}-\mathbf{r}_{2}^{\prime}\right| / c\right)} \cdot
\end{aligned}
$$

$F_{v}^{(+)}$and $F_{v^{\prime}}^{(+)}$are the positive parts of the vacuum incoming beams at $\mathrm{BS}_{2}$ and $\mathrm{BS}_{2}^{\prime}$, respectively.

Now, by taking into account the correlation relations (45) and supposing that $\Delta L_{s} \equiv L_{s, \text { long }}-L_{s \text {, short }}\left(\Delta L_{i} \equiv L_{i \text {, long }}\right.$ $\left.-L_{i, \text { short }}\right)$ is much bigger that the coherence length of the signal (idler) in order to avoid second-order interference, we get

$$
\begin{aligned}
\left|\left\langle F_{s}^{(+)}(\mathbf{0}, t+\tau) F_{i}^{(+)}\left(\mathbf{0}, t+\tau^{\prime}\right)\right\rangle\right| & \frac{1}{4} \mid\left\langle F_{s}^{(+)}\left(\mathbf{0}, t+\tau-\frac{\left|\mathbf{r}_{a}-\mathbf{r}_{2}\right|}{c}-\frac{L_{s, \text { long }}}{c}\right)\right. \\
& \left.\times F_{i}^{(+)}\left(\mathbf{0}, t+\tau^{\prime}-\frac{\left|\mathbf{r}_{b}-\mathbf{r}_{2}^{\prime}\right|}{c}-\frac{L_{i, \text { long }}}{c}\right)\right\rangle \\
& \times e^{i\left(\omega_{s} / c\right)\left(L_{s, \text { long }}+\left|\mathbf{r}_{a}-\mathbf{r}_{2}\right|\right)+i \omega_{i} / c\left(L_{i, \text { long }}+\left|\mathbf{r}_{b}-\mathbf{r}_{2}^{\prime}\right|\right)} \\
& +\left\langle F_{s}^{(+)}\left(\mathbf{0}, t+\tau^{-} \frac{\left|\mathbf{r}_{a}-\mathbf{r}_{2}\right|}{c}-\frac{L_{s, \text { short }}}{c}\right)\right. \\
& \left.\times F_{i}^{(+)}\left(\mathbf{0}, t+\tau^{\prime}-\frac{\left|\mathbf{r}_{b}-\mathbf{r}_{2}^{\prime}\right|}{c}-\frac{L_{i, \text { short }}}{c}\right)\right\rangle \\
& \times e^{i\left(\omega_{s} / c\right)\left(L_{s, \text { short }}+\left|\mathbf{r}_{a}-\mathbf{r}_{2}\right|\right)+i\left(\omega_{i} / c\right)\left(L_{i, \text { short }}+\left|\mathbf{r}_{b}-\mathbf{r}_{2}^{\prime}\right|\right) \mid}
\end{aligned}
$$

where we have taken into account that the fields $F_{v}^{(+)}$and $F_{v^{\prime}}^{(+)}$are uncorrelated among themselves and with $F_{s}^{(+)}$and $F_{i}^{(+)}$. We finally obtain

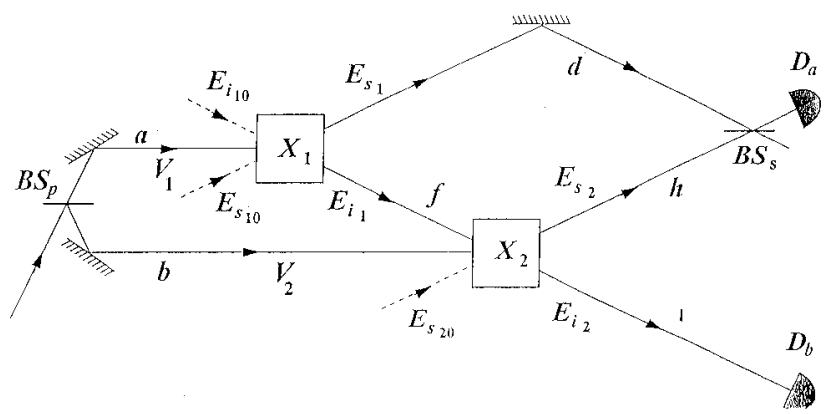

FIG. 5. Experiment of induced coherence without induced emission.

$$
\begin{aligned}
P_{a b}\left(\Delta L_{s}, \Delta L_{i}\right)= & \frac{\eta^{2}}{4 \hbar^{2} \omega_{s} \omega_{i}} g^{2}|V|^{2} \int_{0}^{w} d \tau \int_{0}^{w} d \tau^{\prime}\left\{\left|\nu\left(\tau-\tau^{\prime}\right)\right|^{2}\right. \\
& +\left|\nu\left(\tau^{\prime}-\tau+\frac{\Delta L_{i}-\Delta L_{s}}{c}\right)\right|^{2} \\
& -2 \operatorname{Re}\left[\nu\left(\tau-\tau^{\prime}\right) \nu^{*}\left(\tau^{\prime}-\tau+\frac{\Delta L_{i}-\Delta L_{s}}{c}\right)\right. \\
& \left.\left.\times e^{(i / c)\left(\omega_{s} \Delta L_{s}+\omega_{i} \Delta L_{i}\right)}\right]\right\}
\end{aligned}
$$

which shows a fourth-order interference. If $\left(\Delta L_{i}-\Delta L_{s}\right) / c$ is small in comparison to the coherence time between signal and idler, it is possible to neglect this term in the argument of $\nu\left(\tau^{\prime}-\tau\right)$ and we get a visibility close to $100 \%$.

\section{INDUCED COHERENCE AND INDISTINGUISHABILITY IN TWO-PHOTON INTERFERENCE}

In 1991 Zhou et al. performed an experiment [14] in which fourth-order interference is observed in the superposition of signal photons from two coherently pumped PDC crystals, when the paths of the idler photons are aligned. The experimental situation is illustrated in Fig. 5, in which two similar nonlinear crystals $X_{1}$ and $X_{2}$ are optically pumped by two mutually coherent, classical pump waves of complex amplitudes $V_{1}=V_{2}=V$, and PDC occurs at both crystals, each with the emission of a signal photon and an idler photon. We look for the joint detection probability in the detectors $D_{a}$ and $D_{b}$ when the trajectories of the two idlers $i_{1}, 2$ are aligned and the path difference between the two signals $s_{1}$ and $s_{2}$ is varied slightly. Fourth-order interference disappears when the idlers are misaligned or separated by a beam stop. This experiment has been qualified as "mind boggling" [15], but the analysis in terms of the Wigner function is straightforward.

To start with, let us express the fields at the detectors $D_{a}$ and $D_{b}$ as a function of the incoming fields on the crystals. If $f, d, h$, and $l$ are the distances of $X_{1}$ to $X_{2}, X_{1}$ to $D_{a}, X_{2}$ to $D_{a}$, and $X_{2}$ to $D_{b}$, respectively, then 


$$
\begin{aligned}
& F_{s}^{(+)}\left(\mathbf{r}_{a}, t\right)= \frac{1}{\sqrt{2}}\left[i F_{s_{1}}^{(+)}\left(\mathbf{r}_{a}, t\right)+F_{s_{2}}^{(+)}\left(\mathbf{r}_{a}, t\right)\right] \\
&= \frac{1}{\sqrt{2}}\left\{i F_{s_{10}}^{(+)}\left(\mathbf{0}_{1}, t-d / c\right) e^{i \omega_{s} d / c}+i g^{2}|V|^{2} J F_{s_{10}}^{(+)}\left(\mathbf{0}_{1}, t-d / c\right) e^{i \omega_{s} d / c}\right. \\
&+i g V G F_{i_{10}}^{(-)}\left(\mathbf{0}_{1}, t-d / c\right) e^{i \omega_{s} d / c}+F_{s_{20}}^{(+)}\left(\mathbf{0}_{2}, t-h / c\right) e^{i \omega_{s} h / c}+g^{2}|V|^{2} \\
& \times J F_{s_{20}}^{(+)}\left(\mathbf{0}_{2}, t-h / c\right) e^{i \omega_{s} h / c}+g V G F_{i_{10}}^{(-)}\left[\mathbf{0}_{1}, t-(f+h) / c\right] e^{i\left(\omega_{s} h-\omega_{i} f\right) / c} \\
&\left.+g^{2}|V|^{2} G G^{*} F_{s_{10}}^{(+)}\left[\mathbf{0}_{1}, t-(f+h) / c\right] e^{i\left(\omega_{s} h-\omega_{i} f\right) / c}\right\}, \\
& F_{i}^{(+)}\left(\mathbf{r}_{b}, t^{\prime}\right)=F_{i_{10}}^{(+)}\left[\mathbf{0}_{1}, t^{\prime}-(l+f) / c\right] e^{i \omega_{i}(l+f) / c}+g V G F_{s_{10}}^{(-)}\left[\mathbf{0}_{1}, t^{\prime}-(l+f) / c\right] e^{i \omega_{i}(l+f) / c} \\
&+ g^{2}|V|^{2} J F_{i_{10}}^{(+)}\left[\mathbf{0}_{1}, t^{\prime}-(l+f) / c\right] e^{i \omega_{i}(l+f) / c}+g V G F_{s_{20}}^{(-)}\left(\mathbf{0}_{2}, t^{\prime}-l / c\right) e^{i \omega_{i} l / c} \\
&+ g^{2}|V|^{2} J F_{i_{10}}^{(+)}\left[\mathbf{0}_{1}, t^{\prime}-(l+f) / c\right] e^{i \omega_{i}(l+f) / c} .
\end{aligned}
$$

By using Eq. (56) to compute the coincidence probability, we get

$$
\begin{aligned}
P_{a b}\left(\mathbf{r}_{a}, t+\tau ; \mathbf{r}_{b}, t+\tau^{\prime}\right)= & K^{\prime} \frac{g^{2}|V|^{2}}{2} \mid e^{i\left[\omega_{s} d / c+\omega_{i}(l+f) / c+\pi / 2\right]}\left\{\left\langleF _ { s _ { 1 0 } } ^ { ( + ) } ( \mathbf { 0 } _ { 1 } , t + \tau - d / c ) G F _ { s _ { 1 0 } } ^ { ( - ) } \left(\mathbf{0}_{1}, t+\tau^{\prime}\right.\right.\right. \\
& \left.-(l+f) / c)\rangle+\left\langle F_{i_{10}}^{(+)}\left(\mathbf{0}_{1}, t+\tau^{\prime}-(l+f) / c\right) G F_{i_{10}}^{(-)}\left(\mathbf{0}_{1}, t+\tau-d / c\right)\right\rangle\right\} \\
& +e^{i\left[\omega_{s} h / c+\omega_{i} l / c\right]}\left\{\left\langle F_{s_{20}}^{(+)}\left(\mathbf{0}_{2}, t+\tau-h / c\right) G F_{s_{20}}^{(-)}\left(\mathbf{0}_{2}, t+\tau^{\prime}-l / c\right)\right\rangle\right. \\
& \left.+\left\langle F_{i_{10}}^{(+)}\left[\mathbf{0}_{1}, t+\tau^{\prime}-(l+f) / c\right] G F_{i_{10}}^{(-)}\left[\mathbf{0}_{1}, t+\tau-(f+h) / c\right]\right\rangle\right\}\left.\right|^{2} .
\end{aligned}
$$

Finally, by using Eqs. (44) and (57), the resulting coincidence probability is

$$
\begin{aligned}
P_{a b}= & \frac{\eta^{2} g^{2}|V|^{2}}{8 \hbar^{2} \omega_{s} \omega_{i}} \int_{0}^{\tau} d \tau \int_{0}^{\tau} d \tau^{\prime}\left\{\left|\nu\left(\tau-\tau^{\prime}+l / c+f / c-d / c\right)\right|^{2}\right. \\
& +\left|\nu\left(\tau-\tau^{\prime}+l / c-h / c\right)\right|^{2}-\operatorname{Im}\left[\nu \left(\tau-\tau^{\prime}+l / c+f / c\right.\right. \\
& \left.\left.-d / c) \nu^{*}\left(\tau-\tau^{\prime}+l / c-h / c\right) e^{i\left[\omega_{s}(d-h)+\omega_{i} f\right] / c}\right]\right\}, \quad(75)
\end{aligned}
$$

which, as expected, shows an intensity-intensity interference. As in the experiments of the previous sections, the visibility may be made close to $100 \%$ by making the signal and idler path lengths coincide to within their coherence lengths.

\section{CONCLUSION}

We have developed the Wigner representation formalism in a fashion suitable for the study of experiments involving
PDC photon pairs. The results agree with those obtained using the more common Hilbert-space formulation, both being just two equivalent forms of quantum optics.

The Wigner representation is specially suited for PDC because the Wigner function is Gaussian and positive definite in this case. The Gaussian character simplifies notably the calculation of autocorrelations and cross correlations for the various fields involved. On the other hand, positivity makes possible a picture in terms of pure waves during the propagation, which may aid the intuition in the study of these experiments. However, the detection problem remains and this prevents a straightforward interpretation only with waves. Also the role played by the vacuum field is stressed in this representation.

\section{ACKNOWLEDGMENT}

We acknowledge financial support of CAICYT Project No. PB-95-0594 (Spain).
[1] M. Horne, A. Shimony, and A. Zeilinger, in Quantum Coherence, edited by J. S. Anandan (World Scientific, Singapore, 1990), pp. 356-372.

[2] D. C. Burnham and D. L. Weinberg, Phys. Rev. Lett. 25, 84 (1970).
[3] C. K. Hong, Z. Y. Ou, and L. Mandel, Phys. Rev. Lett. 59, 2044 (1987).

[4] C. K. Hong and L. Mandel, Phys. Rev. A 31, 2409 (1985).

[5] See, e.g., D. F. Walls and G. J. Milburn, Quantum Optics (Springer-Verlag, Berlin, 1994), Chap. 9. 
[6] A vacuum fluctuation argument providing physical insight into photon-photon correlations in experiments with parametric down-converted light have been put forth by M. O. Scully and U. W. Rathe, Opt. Commun. 110, 373 (1994).

[7] A. Casado, T. W. Marshall, and E. Santos, J. Opt. Soc. Am. B 14, 494 (1997)

[8] M. Hillery, R. F. O'Connell, M. O. Scully, and E. P. Wigner, Phys. Rep. 106, 121 (1984).

[9] See, e.g., L. E. Ballentine, Quantum Mechanics (Prentice-Hall, Englewood Cliffs, NJ, 1990), Chap. 17.
[10] R. Ghosh, C. K. Hong, Z. Y. Ou, and L. Mandel, Phys. Rev. A 34, 3962 (1986).

[11] J. G. Rarity and P. R. Tapster, Phys. Rev. Lett. 64, 2495 (1990).

[12] J. D. Franson, Phys. Rev. Lett. 62, 2205 (1989).

[13] P. R. Tapster, J. G. Rarity, and P. C. M. Owens, Phys. Rev. Lett. 73, 1923 (1994).

[14] X. Y. Zou, L. J. Wang, and L. Mandel, Phys. Rev. Lett. 67, 318 (1991).

[15] D. M. Greenberger, M. A. Horne, and A. Zeilinger, Phys. Today 46 (8), 22 (1993). 